\title{
BOUNDARY CONDITIONS INTERPRETED AS CONFORMAL TRANSFORMATION
}

\author{
B. R. SETH
}

1. Introduction. A number of methods exist to solve two-dimensional problems of Dirichlet or Neumann in elasticity, hydrodynamics, and other branches of applied mathematics. Conformal transformation is often used. The analytic function theory method ${ }^{1}$ and the function space method [6] have been recently developed by a number of workers. When the boundary problem is mathematically intractable the soap film method and Southwell's relaxation method are used.

In a two-dimensional harmonic problem the boundary condition amounts to a conformal mapping. The solution of such a problem can be put in the form

$$
z=f(w), \quad z=x+i y, \quad w=\phi+i \psi .
$$

In terms of $\psi$ let the boundary condition be

$$
\begin{aligned}
\psi & =F(x, y) \\
& =x(\phi, \psi) .
\end{aligned}
$$

Thus the solution in (1) is the conformal mapping of the bounded region in the $z$-plane, into a region in the $w$-plane, bounded by the curve in (2). ${ }^{2}$ For example, in a stream line flow bounded by the positive directions of the $x$ - and $y$-axes the boundary condition $\psi=0$ shows that the area in the $z$-plane should be transformed into the upper half of the w-plane. Schwarz-Christoffel transformation at once gives the corresponding result as

$$
\frac{d z^{*}}{d w}=\frac{1}{2 A} w^{1 / 2}, \quad \text { or } \quad w=A z^{2} .
$$

Similarly all problems involving free stream lines and irrotational flow either inside or outside a rectilinear polygonal cylinder can be solved by transforming the boundary condition into a form such that

Presented to the Society, April 30, 1949; received by the editors June 17, 1949 and, in revised form, July 30, 1949 and September 27, 1949.

${ }^{1}$ For an extensive list of references to recent work utilizing this method see [12, $1,13,3]$. Numbers in brackets refer to the references cited at the end of the paper.

${ }^{2}$ It is understood that a one-to-one correspondence exists between the areas in the $z$ - and $w$-planes. 
it amounts to a conformal mapping $[7,8,4]$. This method has been applied to a number of torsion and flexure problems in elasticity by $\mathrm{E}$. Trefftz [14] and B. R. Seth $[9,10]$, and can be easily extended to a number of allied problems. Seth [11] has also shown how it may be used for areas bounded by confocal parabolas. It is proposed to show how it can be of use in general application.

2. Solution for rectilinear boundaries. To begin with let us assume that the boundary is rectilinear and, in terms of $\psi$, the boundary condition is

$$
\frac{\partial \psi}{\partial s}=\left(\sum_{r=0}^{n} x_{r}\right) \cos (y n)-\left(\sum_{r=0}^{n} f_{r}\right) \cos (x n),
$$

where $f_{r}, x_{r}$ are homogeneous integral polynomials of the $r$ th degree in $x$ and $y, n$ is the direction of the normal drawn to the boundary, and $s$ is measured along the boundary.

If the $z$-figure has $m$-angular points and $\left(\xi_{1}, \xi_{2}, \cdots, \xi_{m}\right)$ are the points on the $\xi$-axis in a $\zeta$-plane that correspond to the angular points of the $z$ - and $\Omega_{n+1}$-figures, and $\pi a_{1}, \pi a_{2}, \cdots, \pi a_{m}$ are the internal angles at these points, the solution of the problem can be put in the form $[7,8,4]$

$$
\begin{aligned}
z & =A \int \prod_{r=1}^{m}\left(\zeta-\xi_{r}\right)^{a_{r}-1} d \zeta+B \\
\frac{d^{n+1} w}{d z^{n+1}} & =\frac{d^{n+1}(\phi+i \psi)}{d z^{n+1}}=\Omega_{n+1} \\
& =c \int R(\zeta) \prod_{r=1}^{m}\left(\zeta-\xi_{r}\right)^{-(n+1) a_{r}} d \zeta+D \\
\Omega_{n+1} & =X_{n+1}+c Y_{n+1},
\end{aligned}
$$

$R(\zeta)$ being a rational integral function of the $[(n+1) m-2(n+2)]$ th degree with real coefficients.

If the $z$-area extends to infinity in all directions and is internally bounded by a polygon, (4) should be replaced by $[5,2]$

$$
\begin{gathered}
z=A \int \prod_{r=1}^{m} \frac{\left(\zeta-\xi_{r}\right)^{a_{r}-1}}{\left(1+\zeta^{2}\right)^{2}} d \zeta+B, \\
\sum_{r=1}^{m} \frac{\xi_{r}\left(a_{r}-1\right)}{1+\xi_{r}^{2}}=0, \quad \sum_{r=1}^{m} \frac{a_{r}-1}{1+\xi_{r}^{2}}=1,
\end{gathered}
$$

and $R(\zeta)$ in (5) is now a rational integral function of $[(n+1) m+2 n]$ th 
degree with real coefficients. The boundary condition (3) takes the form

$$
\begin{aligned}
& X_{n+1} \sin (n+1) \alpha_{p}+Y_{n+1} \cos (n+1) \alpha_{p} \\
& \quad=L^{n}\left[\sin \alpha_{p} f_{n}\left(\cos \alpha_{p}, \sin \alpha_{p}\right)+\cos \alpha_{p} \chi_{n}\left(\cos \alpha_{p}, \sin \alpha_{p}\right)\right] \\
& \quad(p=1,2, \cdots, m) .
\end{aligned}
$$

3. Curved boundaries. To extend this method to curved boundaries let us assume that the transformation

$$
z=g(t)
$$

maps the region with curvilinear boundary in the $z$-plane onto a region with rectilinear boundary in the $t$-plane. The corresponding boundary condition can be written down with the help of (3). In general $\sum f_{r}$ and $\sum x_{r}$ will be infinite series. For confocal parabolas the transformation is

$$
z=t^{2}
$$

and the problem can be readily solved. In other cases we take the first $n$ terms and solve the problem. Making $n \rightarrow \infty$ gives the desired solution.

To illustrate this method we take the torsion problem of an elliptic cylinder. The boundary condition is

$$
\psi=2^{-1}\left(x^{2}+y^{2}\right)+\text { a constant }
$$

which in elliptic coordinates

$$
z=c \cosh t=c \cosh (\alpha+i \beta)
$$

becomes

$$
\psi=4^{-1} c^{2} \cos 2 \beta+\text { a constant }
$$

$$
=4^{-1} c^{2}\left[1-\frac{(2 \beta)^{2}}{2 !}+\frac{(2 \beta)^{4}}{4 !}-\cdots+(-1)^{n} \cdot \frac{(2 \beta)^{2 n}}{2 n !}\right]
$$

if powers of $\beta$ higher than the $2 n$th are neglected.

The boundary condition (6.3) takes the form

$$
X_{2 n} \sin 2 n \alpha_{p}+Y_{2 n} \cos 2 n \alpha_{p}=4^{-1} c^{2}(-1)^{n}\left(2 \sin \alpha_{p}\right)^{2 n},
$$

which shows that $\Omega_{2 n}$-figure is the point

$$
\Omega_{2 n}=4^{-1} i c^{2} 2^{2 n} \text {. }
$$

Integrating this $2 n$ times in succession, and using the boundary condition corresponding to (16) at each stage, we get 


$$
\begin{aligned}
w= & \frac{1}{4} c^{2} i\left[\frac{(2 t)^{2 n}}{2 n !}+\frac{(2 t)^{2 n-2}}{(2 n-2) !}+\cdots+\frac{(2 t)^{2}}{2 !}+1\right] \\
& +\frac{1}{4} c^{2} i c_{0}\left[\frac{(2 t)^{2 n-2}}{(2 n-2) !}+\frac{(2 t)^{2 n-4}}{(2 n-4) !}+\cdots+\frac{(2 t)^{2}}{2 !}+1\right],
\end{aligned}
$$

where $c_{0}$ is to be adjusted to satisfy (11.1).

Making $n \rightarrow \infty$ we get

$$
w=4^{-1} c^{2} i \cosh 2 t\left(1+c_{0}\right) .
$$

Using (11.1) we get $1+c_{0}=\operatorname{sech} 2 \alpha$. Substituting the value of $\cosh 2 t$ from (10) we get the known result.

\section{REFERENCES}

1. R. C. F. Bartels, Trans. Amer. Math. Soc. vol. 53 (1943) pp. 1-3.

2. W. G. Bickley, Philos. Trans. Roy. Soc. London Ser. A vol. 228 (1929) pp. 235-274.

3. S. Ghosh, Bull. Calcutta Math. Soc. vol. 40 (1948) pp. 107-115.

4. J. Hodgkinson, Quart. J. Math. Oxford Ser. vol. 6 (1935) pp. 171-183.

5. J. Kluyver, Zeitschrift für Mathematische Physik vol. 40 (1895) pp. 129-150.

6. W. Prager and J. L. Synge, Quarterly of Applied Mathematics vol. 5 (1947) pp. 241-269.

7. B. R. Seth, Quart. J. Math. Oxford Ser. vol. 5 (1934) pp. 161-171.

8. - The Philosophical Magazine vol. 20 (1935) pp. 632-640.

9. - Proc. Cambridge Philos. Soc. vol. 33 (1934) pp. 392-403.

10. — The Philosophical Magazine vol. 22 (1936) pp. 582-598; vol. 23 (1937) pp. 745-757. 453.

11. - Proceedings of the Indian Academy of Sciences vol. 9 (1939) pp. 447-

12. I. S. Sokolnikoff, Bull. Amer. Math. Soc. vol. 48 (1942) pp. 539-555.

13. A. C. Stevenson, The Philosophical Magazine vol. 39 (1948) p. 300.

14. E. Trefftz, Math. Ann. vol. 82 (1921) pp. 97-112.

Iowa State College of Agriculture and Mechanic Arts 